\title{
FIGO Stage IIIA1(i)
}

National Cancer Institute

\section{Source}

National Cancer Institute. FlGO Stage IIIA1(i). NCI Thesaurus. Code C128103.

A FIGO stage term that applies to ovarian cancer, fallopian tube cancer, and primary peritoneal cancer. It refers to cancer with positive retroperitoneal lymph nodes only. Metastasis equal or less than $10 \mathrm{~mm}$. 